\title{
The ticking clock: does actively making an enhanced care team aware of the passage of time improve pre-hospital scene time following traumatic incidents?
}

\author{
L. Curtis ${ }^{1 *}$, E. ter Avest ${ }^{1,2}$, J. Griggs ${ }^{1}$, J. Wiliams ${ }^{3,4}$ and R. M. Lyon ${ }^{1,3}$
}

\begin{abstract}
Introduction: Pre-hospital enhanced care teams like Helicopter Emergency Medical Services (HEMS) are often dispatched to major trauma patients, including patients with traumatic brain injuries and those with major haemorrhage. For these patients, minimizing the time to definitive care is vital. The aim of this study was to determine whether increased awareness of elapsed on scene time produces a relevant time performance improvement for major trauma patients attended by HEMS, and weather introducing such a timer was feasible and acceptable to clinicians.

Methods: We performed a prospective cohort study of all single casualty traumatic incidents attended by Air Ambulance Kent Surrey Sussex (AAKSS) between 15 October 2016 and 23 May 2017 to test if introduction of a prompting scene timer within the service resulted in a reduction in pre-hospital scene times.

Results: The majority of the patients attended were male (74\%) and sustained blunt trauma (92\%). Overall, median scene time was 25.5 [IQR16.3] minutes before introduction of the scene timer and 23.0 [11.0] minutes after introduction, $p=0.13$ ). Scene times for patients with a GCS $<8$ and for patients requiring prehospital anaesthesia were significantly lower after introduction of the timer (28 [IQR 14] vs 25 [1], $p=0.017$ and 34 [IQR 13] vs 28 [IQR11] minutes, $p=0.007$ respectively). The majority of clinicians felt the timer made them more aware of passing time (91\%) but that this had not made a difference to scene time (62\%) or their practice (57\%).

Conclusion: Audible scene timers may have the potential to reduce pre-hospital scene time for certain single casualty trauma patients treated by a HEMS team, particularly for those patients needing pre-hospital anaesthesia. Regular use of on-scene timers may improve outcomes by reducing time to definitive care for certain subgroups of trauma patients.
\end{abstract}

Keywords: Trauma, HEMS, Scene time, Prompting timer

\footnotetext{
* Correspondence: LeighC@aakss.org.uk

${ }^{1}$ Air Ambulance Kent, Surrey and Sussex, Redhill Aerodrome, Redhill Airfield, Redhill, Surrey RH1 5YP, UK

Full list of author information is available at the end of the article
}

C C The Author(s). 2020 Open Access This article is licensed under a Creative Commons Attribution 4.0 International License, which permits use, sharing, adaptation, distribution and reproduction in any medium or format, as long as you give appropriate credit to the original author(s) and the source, provide a link to the Creative Commons licence, and indicate if changes were made. The images or other third party material in this article are included in the article's Creative Commons licence, unless indicated otherwise in a credit line to the material. If material is not included in the article's Creative Commons licence and your intended use is not permitted by statutory regulation or exceeds the permitted use, you will need to obtain permission directly from the copyright holder. To view a copy of this licence, visit http://creativecommons.org/licenses/by/4.0/ The Creative Commons Public Domain Dedication waiver (http://creativecommons.org/publicdomain/zero/1.0/) applies to the data made available in this article, unless otherwise stated in a credit line to the data. 


\section{Background}

In many western countries, trauma is the leading cause of death in those under 40 years of age [2]. For several decades one of the corner stone principals of prehospital trauma care has been to improve survival by reducing the time taken to deliver patients from the point of injury to definitive care ("scoop and run"). Studies attempting to explore and establish the relationship between pre-hospital time and patient outcome (mortality) in the civilian setting have shown largely equivocal results $[3,4]$. However, a clear mortality reduction associated with shorter scene times has been demonstrated for more discreet trauma cohorts, such as patients suffering from penetrating trauma [5-7] and traumatic brain injury (TBI) $[8,9]$.

Enhanced pre-hospital care teams, such as Helicopter Emergency Medical Services (HEMS), are often dispatched to major trauma patients, including patients with TBI and patients with penetrating injuries. Enhanced care teams have the ability to perform critical care interventions at the scene ("stay and play") beyond the capability of other pre-hospital responders. However, performing enhanced pre-hospital interventions can increase scene time, especially when advanced interventions, such as rapid sequence induction (RSI) have to be performed [1, 10-12]. Close monitoring of scene times is therefore advisable, especially as clinicians generally tend to underestimate the time elapsed whilst performing complex clinical interventions [13, 14].

Previous studies have shown that temporal awareness improves performance [15] and reduces the time taken to deliver clinical interventions [16]. One way to improve temporal awareness, is through the use of a prompting timer. The aim of this study was to determine whether the introduction of an audible pre-hospital scene timer, which alerted clinicians to elapsed time on scene, produced a relevant time performance improvement, and weather introducing such a timer was feasible and acceptable to clinicians.

\section{Methods}

\section{Study design}

We performed a prospective cohort study of all single casualty traumatic incidents attended by Air Ambulance Kent, Surrey Sussex (AAKSS) between 15 October 2016 and 23 May 2017, to test if making a HEMS team actively aware of the passage of time using a prompting scene timer does improve the prehospital scene time of traumatic incidents.

To test the hypothesis that making a HEMS team actively aware of the passage of time does improve the pre-hospital times of traumatic incidents, we performed a pre- and post change evaluation of the introduction of a prompted scene timer. Based on previous publications
[15] and consensus opinion from within the service, an observed effect size in excess of $15 \%$ was felt to be indicative of significant change. In order to detect this with a power of $80 \%$, and a mean pre-intervention scene time of $25 \pm 11$ min our sample size was set at $>125$ patients in each group. Subgroup analysis was pre-specified for potentially confounding factors influencing scene time (age, mechanism of injury, GCS, interventions on scene, mode of transport HEMS and time of day).

\section{Setting}

AAKSS is a Helicopter Emergency Medical Service (HEMS) covering three counties $\left(9000 \mathrm{~km}^{2}\right)$ in the southeast of the UK with a resident population of approximately 4.6 million people. This service operates 24 $\mathrm{h}$ a day and its clinical team consists of a doctor and paramedic. Paramedics have several years of critical care experience, and doctors are in their last stage of specialty training or consultant level. Both doctors and paramedics follow an intensive prehospital induction course, and have a supervised sign-off period before they start working independently. HEMS is deployed to patients suffering suspected critical, or life threating injury or illness. Annually, this service responds to in excess of 2500 incidents.

\section{Intervention}

After an initial period wherein scene times were measured without feedback to the crews, a prompting timer was introduced. The timer system involved was a GYMBOSS interval timer being placed on the services' dispatch desk. The timer was started by the dispatcher, once notified by the HEMS team that they had arrived on scene. After every $5 \mathrm{~min}$ the GYMBOSS sounded an alarm prompting the dispatcher to make a radio call to the HEMS team stating the time elapsed. The process continued until the team departed the scene with the patient.

\section{Study population}

Incidents were selected for inclusion using a consecutive sampling strategy of all single casualty incidents involving traumatic injury attended by the service during the project period. Incidents where patients required extrication were excluded, as the process was beyond the direct control of the HEMS team and inclusion would potentially confound the true effect of the time. Incidents where the HEMS team did not convey the patient from scene to hospital, were also excluded, as it was not feasible to establish scene times for these patients. Finally, a scene time of less than 5 min was an exclusion criterium, as scene times below $5 \mathrm{~min}$ could not be affected by a 5 min interval timer. 


\section{Outcome measures}

The primary outcome was defined as the time spend on scene, from arrival on the patients' side until leaving scene.

The secondary outcome was the feasibility and acceptability of using the prompting scene timer as expressed on a 5-point scale.

\section{Data acquisition}

For both pre- and post-implementation periods timings were collected and entered real-time in the electronic patient clinical record system (HEMSbase 2.0, Medic One Systems Ltd., UK) by the HEMS dispatcher in the ambulance control centre. Information about potential confounders affecting scene time was collected for both periods from HEMSbase. The following data were retrieved: Age, mechanism of injury [road traffic collision/ other], patient GCS, interventions on scene [prehospital anesthesia/thoracostomy/thoracotomy/blood product administration], mode of transport [car/helicopter] and time of day [day/night].

At the conclusion of the post change period, all HEMS-team members were contacted and invited to complete an anonymous electronic 10-question structured questionnaire to determine the effectiveness, perception and user acceptability of the scene timers (Supplementary file 1).

\section{Ethical considerations}

This project met the National Research Ethics Service (NRES) definition of service evaluation audit (NRES, 2009) and therefore did not require ethical approval. Ethics approval however was sought and was granted for the conduct of the staff survey by the University of Hertfordshire Research Ethics Committee.

\section{Statistical analysis}

Shapiro Wilk tests were performed to assess normal distribution and residual plots were drawn to assess linearity of data. Descriptive statistics are given as numbers [\%] or median [IQR]. Comparisons across groups for baseline characteristics were made using Fisher's exact test and Mann-Whitney $\mathrm{U}$ test where appropriate. Scene-time comparisons were made by Mann-Whitney $\mathrm{U}$ test or independent $\mathrm{t}$-test where appropriate. Per protocol scene time comparisons were made using univariate analysis of variance (GLM) with mode of transportation (helicopter/ambulance) as a co-factor, with pre-specified subgroup analysis for potentially confounding factors. Survey data was summarised using descriptive statistics. Missing values are reported in the results section of the manuscript according to the STROBE guideline [17]. A $p$-value $<0.05$ was regarded as statistically significant. All statistical analyses were conducted using IBM SPSS 23.0 for Windows statistical package.

\section{Results \\ Study population}

During the study period, a total of 858 patients were attended, of which 594 (69\%) met the inclusion criteria. 54 patients $(9 \%)$ who required extrication were excluded, as were 237 (40\%) patients who were not conveyed from scene by the HEMS team. 16 patients $(3 \%)$ had a scene time less than $5 \mathrm{~min}$, and for 5 patients (1\%) scene times were either missing or erroneous (Fig. 1). Scene time data were captured for a total of 282 patients: 134 patients in the period before introduction of the scene timer, and 148 in the period after introduction. For 5 patients in the post-introduction group the protocol was violated and the timer had not been started. These were excluded from the further (per protocol) analysis.

Table 1 shows the study population characteristics. Before- and after introduction of the scene timer, the majority of patients was male, involved in RTC's- or accidental injury resulting, and sustained resulting blunt trauma. Frequency distribution of mechanism of injury, type of injury, moment of injury, and interventions performed on scene was equal for both study periods. In the period after introduction of the scene timer however, significantly more patients were transported to hospital by helicopter compared to by ground ambulance ( $37 \%$ vs $59 \%, p<0.001$ ). As overall scene time for patients transported to hospital by helicopter was longer than for those being transported by road $(25$ $\min$ [IQR 12.3] vs $22 \mathrm{~min}$ [15.2], $p<0.001$ ), mode of transport was identified as a relevant co-variate for subsequent analysis of variance of scene time.

\section{Effect of prompted timer}

Univariate analysis of variance was performed with mode of transportation as a co-factor to investigate the effect of the prompted timer on scene times. Overall median scene times were slightly lower after implementation of the prompted timer (23.0 [IQR 11.0] minutes vs 25.5 [IQR 16.3]). However, the difference did not meet the prespecified clinically relevant difference of $15 \%$, and statistical significance was not reached ( $p=0.128)$, Table 2 .

Subgroup analysis demonstrated a significant difference in scene time for patients who underwent prehospital anaesthesia and for patients with a low GCS. For these teams attending these patients, the prompted timer was associated with a reduction in scene time of respectively $6 \mathrm{~min}$ (34 [IQR 13] vs 28 [IQR11] minutes, $p=$ $0.007)$ and $3 \mathrm{~min}$ (28 [IQR 14] vs 25 [10] minutes, $p=$ $0.017)$, Table 2. 


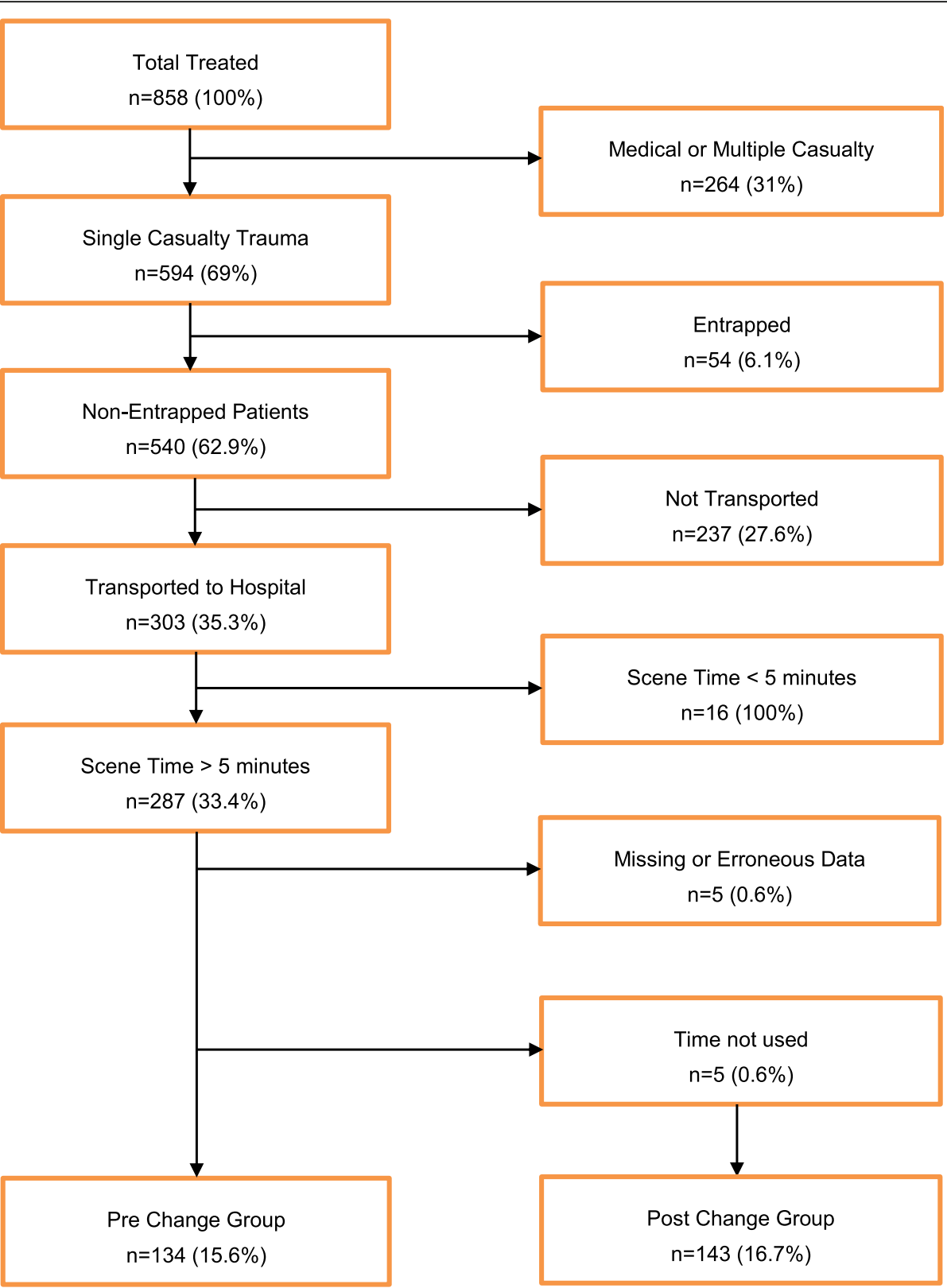

Fig. 1 Inclusion Criteria

\section{Feasibility and acceptability}

Of the 45 clinicians who took part in the project (doctors $n=25$, paramedics $n=20$ ) anonymised survey responses were received from 21 (doctors $n=10$, paramedics $n=11$ ) giving a response rate of $47 \%$ overall.

All respondents reported that they were aware of the audible time calls at least some of the time, the majority (67\%) describing this as "often". The vast majority of clinicians (91\%) agreed that the time calls had made them more aware of the passage of time on scene. Despite this, the majority felt this had not made a difference to scene times $(62 \%)$ or changed their practice in any way (57\%). Of the 9 who did feel their practice had changed $89 \%(n=8)$ described this change with some positive level of acceptability.

Acceptability of the scene timer was viewed as either "somewhat" or "very acceptable" by $72 \%$ of all respondents, however $10 \%(n=2)$ did find the timer "somewhat unacceptable". 76\% $(n=16)$ described having experienced some degree of timer-related distraction. Despite this only 2 respondents (10\%) expressed the view that they would not want the use of the timer to continue. 
Table 1 Characteristics of the study population

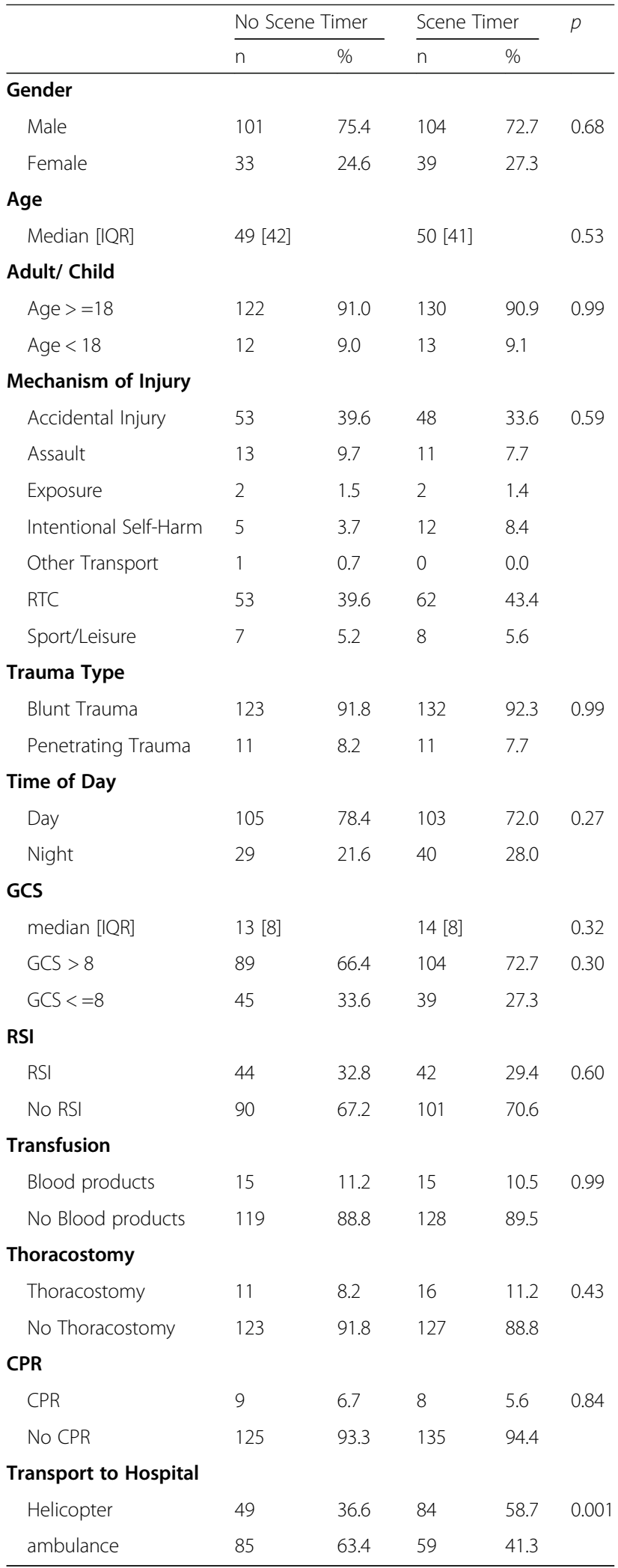

GCS Glasgow Coma Scale, $R S I$ Rapid sequence induction, $C P R$ cardiopulmonary resuscitation

\section{Discussion}

Introduction of an audible scene timer into a HEMS service was associated with a statistically significant reduction in scene time for patients who require prehospital anaesthesia or have GCS $<=8$.

Experimental psychology has extensively studied the human perception of time and shown that perceived duration of time is affected by whether an individual is aware of the passage of time [18]. Research has shown that the less cognitive resource focussed on monitoring time, the shorter the period is perceived [18]. This same phenomenon has been demonstrated in clinical settings where clinicians have been found to significantly underestimate the amount of time elapsed whilst performing complex clinical interventions $[13,14]$.

We did not demonstrate a positive effect of prompting scene times for all undifferentiated single casualty trauma incidents. Although there was an overall trend towards lower scene times, specifically for blunt trauma, statistical significance was not reached. This may have various reasons. First, time awareness of clinicians is especially affected when their attention is focussed on (complex) clinical interventions [13, 14]. Therefore, it is likely that the largest effect of time prompting is to be expected in the subgroup of patients who undergo a prehospital intervention (such as prehospital anaesthesia). Second, besides time awareness, scene time is dependent on many other factors. Severity of injuries and resultant perceived urgency may play a role as well, as scene time has been shown to be longer for less injured patients $[19,20]$. As we have not quantified perceived injury severity, this may have been a confounding factor. Finally, mode of transport to hospital was identified as a significant confounding factor, with longer scene times for patients transported by helicopter (often needing a secondary transport from scene to landing site). Although mode of transportation was entered as a cofactor in the pre- and post-intervention comparisons, we cannot completely exclude that this has affected our results, as mode of transport may also reflect differences in injury severity.

The tenant that time is a critical factor appears to have originated largely from expert opinion [21] and popularised by research findings from the Vietnam War where the $2 \%$ increase in survival compared to previous conflicts was attributed to reducing time to definitive care to 1 "golden" hour [22]. Although several studies have failed to substantiate the existence of a time and survival relationship for the undifferentiated and blunt civilian trauma cohorts [7, 23-25], this relationship has been established for patients with penetrating injuries, and patients with TBI [5-9].

In our study, we found a significant reduction is scene time $(-10.7 \%)$ for patients with a GCS $<8$ and a similar 
Table 2 Effect of a prompting scene timer on scene times for single casualty traumatic incidents attended by HEMS

\begin{tabular}{|c|c|c|c|c|c|c|c|c|c|}
\hline \multirow[t]{2}{*}{ Group } & \multicolumn{3}{|c|}{ No Scene Timer } & \multicolumn{3}{|c|}{ Scene Timer } & \multirow{2}{*}{$\begin{array}{l}\text { Difference } \\
\text { in mins. }\end{array}$} & \multirow[t]{2}{*}{$\%$ Change } & \multirow[t]{2}{*}{ Significance $p$} \\
\hline & Number & Median (Mean) & IQR (SD) & Number & Median (Mean) & IQR (SD) & & & \\
\hline All Incidents & 134 & 25.5 & 16.3 & 143 & 23.0 & 11.0 & 2.5 & 9.8 & 0.13 \\
\hline Adults (age > =18) & 122 & 26.0 & 16.3 & 130 & 23.0 & 11.3 & 3.0 & 11.5 & 0.20 \\
\hline Children (age < 18) & 12 & $(24.4)$ & $(12.1)$ & 13 & $(22.5)$ & $(7.7)$ & 1.9 & 7.7 & 0.45 \\
\hline RTC & 53 & 27.0 & 17.0 & 62 & 23.0 & 13.5 & 4.0 & 14.8 & 0.13 \\
\hline Non-RTC Mechanism & 81 & 25.0 & 18.5 & 81 & 24.0 & 11.0 & 1.0 & 4.0 & 0.27 \\
\hline Blunt Trauma & 123 & 27.0 & 17.0 & 132 & 24.0 & 10.8 & 3.0 & 11.1 & 0.08 \\
\hline Penetrating Trauma & 11 & 8.0 & 5.0 & 11 & 11.0 & 14.0 & -3.0 & -37.5 & 0.64 \\
\hline Day & 105 & 27.0 & 16.0 & 103 & 23.0 & 12.0 & 4.0 & 14.8 & 0.08 \\
\hline Night & 29 & $(24.1)$ & $(13.0)$ & 40 & $(24.9)$ & $(9.1)$ & -0.9 & -3.6 & 0.89 \\
\hline $\mathrm{GCS}>8$ & 89 & 22.0 & 18.0 & 104 & 23.0 & 12.8 & -1.0 & -4.5 & 0.92 \\
\hline $\mathrm{GCS}<=8$ & 45 & $(29.7)$ & $(10.1)$ & 39 & $(25.2)$ & $(8.1)$ & 4.5 & 15.1 & 0.028 \\
\hline RSI & 44 & 34.0 & 13. 0 & 42 & 28.0 & 11.0 & 6.0 & 17.6 & 0.007 \\
\hline No RSI & 90 & 19.0 & 14.3 & 101 & 21.0 & 11.0 & -2.0 & -10.5 & 0.96 \\
\hline Blood products & 15 & $(27.3)$ & $(12.1)$ & 15 & (30.8) & (8.8) & -3.5 & -12.9 & 0.37 \\
\hline No Blood products & 119 & 25.0 & 16.0 & 128 & 23.0 & 11.0 & 2.0 & 8.0 & 0.21 \\
\hline Thoracostomy & 11 & $(32.5)$ & $(15.7)$ & 16 & $(31.9)$ & $(12.5)$ & 0.5 & 1.6 & 0.93 \\
\hline No Thoracostomy & 123 & 25.0 & 17.0 & 127 & 23.0 & 10.0 & 2.0 & 8.0 & 0.25 \\
\hline CPR & 9 & $(26.7)$ & (9.9) & 8 & $(29.1)$ & (13.3) & -2.5 & -9.2 & 0.76 \\
\hline Helicopter Transport & 49 & 27.0 & 13.5 & 84 & 24.0 & 11.0 & 3.0 & 11.1 & 0.28 \\
\hline Ambulance Transport & 85 & 23.0 & 19.0 & 59 & 21.0 & 11.0 & 2.0 & 8.7 & 0.50 \\
\hline
\end{tabular}

RTC road traffic collision, GCS Glasgow coma scale, $R S$ I rapid sequence induction, CPRcardiopulmonary resuscitation;

reduction for patients requiring prehospital anaesthesia $(-17.6 \%)$. As GCS $<8$ is a common finding in TBI and TBI is also a common indication for performing RSI, it is highly likely that a large proportion of the patients in both subgroups will also be TBI patients. As TBI patients as a subset have been found to benefit from reductions in prehospital time $[8,9]$, there is a clear potential for the introduction of the scene timer to improve clinical outcome for these patients.

Minimizing scene times is equally important for patients suffering from penetrating injuries $[5,6]$, as the number of interventions (and thereby the time on scene) in these patients is directly related to their mortality [6]. However, we could not demonstrate an effect of scene time prompting on scene times for these patients, as the number of patients with penetrating injury was small and our study was not powered to detect such difference. Furthermore, scene times for these patients are already relatively short (median $11 \mathrm{~min}$ ), leaving less potential to for any intervention to improve these timings any further.

The use of the timer was found to be acceptable by the majority of clinicians who filled out the questionnaire and a majority felt this had made them more cognitively aware of the passage of time. However, most did not feel that the scene timer had affected their scene time or caused them to change practice. These perceptions are not supported by the actual scene time data describing a trend towards improving scene times. The number of clinicians reporting some degree of distraction is not surprising, as task fixation is common when completing complex tasks $[13,19]$. It is likely that the distraction described is representative of the deliberate cognitive sharing created by the timer. This is supported by the respondents who describe an increased positive focus on time.

\section{Limitations}

Our study has several limitations, most being inherent to the study design. First, in order to be able to compare the groups pre- and post-intervention, a large number of patients (multi casualty, entrapped, not transported) had to be excluded, which limits the generalizability of our study results. Second, analysis was on a per protocol basis, excluding 5 patients in whom the protocol was violated in the post-implementation period. Including these patients (in an intention to treat analysis) however, did not affect our results. Third, the proportion of patients treated by each individual clinician in each group was not evaluated. As individual clinicians vary significantly in 
terms of technical and experiential ability, this could have affected our findings, although the relatively small patient to clinician ratio makes such a performance bias unlikely.

Fourth, although subgroups were specified before data collection, no adjustment of sample size was made and therefore the risk of specifically type 2 error in subgroup analysis cannot be excluded. Further, we did not record efficiency or complication rate of procedures performed, nor did we have information on ISS scores or final outcome of our patients. As introducing a scene timer may put (additional) stress on clinicians on scene, these may have affected their ability to perform procedures as the delivery of prehospital anaesthesia. In addition, the response to the questionnaire was low (47\%), thereby somewhat limiting the conclusions regarding feasibility and acceptability that can be drawn from it. Finally, we recognize that an audible timer may create distraction whilst at the same time it may have acted as a passive observer creating a perceived demand for performance thereby creating attention bias [26]. Whilst little is known of the mechanism and scale of such an "Hawthorne Effect" its existence has been conclusively established [27], and might have affected our findings.

\section{Conclusion}

Audible scene timers may have the potential to reduce pre-hospital scene time for certain single casualty trauma patients treated by a HEMS team, particularly for those patients needing pre-hospital anaesthesia. Regular use of on-scene timers may improve outcomes by reducing time to definitive care for certain subgroups of trauma patients.

\section{Supplementary information}

Supplementary information accompanies this paper at https://doi.org/10. 1186/s13049-020-00726-9.

Additional file 1: Supplementary file 1. Study Questionaire.

\section{Abbreviations}

AAKSS: Air Ambulance Kent Surrey and Sussex; GCS: Glasgow Coma Scale; HEMS: Helicopter emergency Medical service; RSI: Rapid Sequence Induction; RTC: Road traffic collision

\section{Acknowledgements}

The authors want to thank the KSS dispatchers (C Lewis, E Lambert, R Knight, A Sands, M Bushell, N Antino) and the KSS HEMS paramedics and doctors for participating in the study.

\section{Authors' contributions}

LC conceived the study. RL supervised the project as a whole. LC collected the data. LC and EtA analysed the data, and EtA, LC and RL drafted the manuscript. All authors read and approved the final version of the manuscript.

\section{Funding}

No external funding was received for this study. Internal support was provided by South East Coast Ambulance Service NHS Foundation Trust and Kent, Surrey \& Sussex Air Ambulance Trust.

\section{Availability of data and materials}

The datasets used and/or analysed during the current study are available from the corresponding author on reasonable request.

\section{Ethics approval and consent to participate}

This project met National Institute for Healthcare Research (NIHR, UK) criteria for service evaluation and formal ethical approval was therefore not required. The project was approved by the AAKSS Research \& Development

Committee.

\section{Consent for publication}

Not applicable.

\section{Competing interests}

The authors declare that they have no competing interests.

\section{Author details}

${ }^{1}$ Air Ambulance Kent, Surrey and Sussex, Redhill Aerodrome, Redhill Airfield, Redhill, Surrey RH1 5YP, UK. ²Department of Emergency Medicine, University Hospital Groningen, Groningen, the Netherlands. ${ }^{3}$ School of Health Sciences, University of Surrey, Guildford, UK. ${ }^{4}$ South East Coast Ambulance Service NHS Foundation Trust, Crawley, UK.

Received: 19 December 2019 Accepted: 15 April 2020

Published online: 29 April 2020

\section{References}

1. Wyen $\mathrm{H}$, Lefering $\mathrm{R}$, Maegele $\mathrm{M}$, Brockamp $\mathrm{T}$, Wafaisade $\mathrm{A}$, Wutzler $\mathrm{S}$, et al The golden hour of shock - how time is running out: prehospital time intervals in Germany - a multivariate analysis of 15, 103 patients from the TraumaRegister DGU ๑. Emerg Med J. 2013;30(12):1048-55. https://doi.org/ 10.1136/emermed-2012-201962.

2. TARN. Major trauma care in England. London: The Stationery Office; 2010. (February), 4.

3. Feero $S$, Hedges JR, Simmons E, Irwin L. Does out-of-hospital EMS time affect trauma survival? Am J Emerg Med. 1995;13(2):133-5. https://doi.org/ 10.1016/0735-6757(95)90078-0.

4. Sampalis JS, Tamim H, Denis R, Boukas S, Ruest SA, Nikolis A, et al Ineffectiveness of on-site intravenous lines: is prehospital time the culprit? J of Trauma. 1997;43(4):608-15. https://doi.org/10.1097/00005373-19971000000008.

5. Funder KS, Petersen JA, Steinmetz J. On-scene time and outcome after penetrating trauma: an observational study. Emerg Med J. 2011;28(9):797801. https://doi.org/10.1136/emi.2010.097535.

6. Kidher E, Krasopoulos G, Coats T, Charitou A, Magee P, Uppal R, Athanasiou $\mathrm{T}$. The effect of prehospital time related variables on mortality following severe thoracic trauma. Injury. 2012;43(9):1386-92. https://doi.org/10.1016/j. injury.2011.04.014

7. McCoy CE, Menchine M, Sampson S, Anderson C, Kahn C. Emergency medical services out-of-hospital scene and transport times and their association with mortality in trauma patients presenting to an urban level i trauma center. Ann Emerg Med. 2013;61(2):167-74. https://doi.org/10.1016/j. annemergmed.2012.08.026.

8. Dinh MM, Bein K, Roncal S, Byrne CM, Petchell J, Brennan J. Redefining the golden hour for severe head injury in an urban setting: the effect of prehospital arrival times on patient outcomes. Injury. 2013;44(5):606-10. https://doi.org/10.1016/j.injury.2012.01.011.

9. Tien HCN, Jung V, Pinto R, Mainprize T, Scales DC, Rizoli SB. Reducing timeto-treatment decreases mortality of trauma patients with acute subdural hematoma. Ann Surg. 2011;253(6):1178-83. https://doi.org/10.1097/SLA. ob013e318217e339.

10. van der Velden MWA, Ringburg AN, Bergs EA, Steyerberg EW, Patka P Schipper IB. Prehospital interventions: time wasted or time saved? An observational cohort study of management in initial trauma care. Emerg Med J. 2008;25(7):444-9. https://doi.org/10.1136/emj.2007.052662.

11. Carr BG, Brachet T, David G, Duseja R, Branas CC. The time cost of Prehospital intubation andlntravenous access in trauma patients. Prehosp Emerg Care. 2008:12(3):327-32. https://doi.org/10.1080/10903120802096928.

12. Cudnik MT, Newgard CD, Wang H, Bangs $C$, Herringtion R. Endotracheal intubation increases out-of-hospital time in trauma patients. Prehosp Emerg Care. 2007;11(2):224-9. https://doi.org/10.1080/10903120701205208. 
13. Cemalovic N, Scoccimarro A, Arslan A, Fraser R, Kanter M, Caputo N. Human factors in the emergency department: is physician perception of time to intubation and desaturation rate accurate? Emerg Med Australas. 2016. https://doi.org/10.1111/1742-6723.12575.

14. Trevisanuto D, De Bernardo G, Res G, Sordino D, Doglioni N, Weiner G, Cavallin F. Time perception during neonatal resuscitation. J Pediatr. 2016. https://doi.org/10.1016/j.jpeds.2016.07.003.

15. Grosjean $V$, Terrier P. Temporal awareness: pivotal in performance? Ergonomics. 1999;42(11):1443-56. https://doi.org/10.1080/001401399184802.

16. Rosini J, Ciehanowski M, Nomura J, Shulkov S, Munson A, Raser-Schramm J. A before-after study evaluating use of a tPA timer on door-to-needle times in acute ischemic stroke. Crit Care Med. 2013;41(12):A109.

17. von Elm E, Altman DG, Egger M, Pocock SJ, Gøtzsche PC. Vandenbroucke JP; STROBE initiative. The strengthening the reporting of observational studies in epidemiology (STROBE) statement: guidelines for reporting observational studies. Int J Surg. 2014;12:1495-9.

18. Brown SW. Attentional resources in timing: interference effects in concurrent temporal and nontemporal working memory tasks. Percept Psychophys. 1997;59(7):1118-40. https://doi.org/10.3758/BF03205526.

19. Goodacre SW, Gray A, McGowan A. On-scene times for trauma patients in West Yorkshire. J Accid Emerg Med. 1997;14(5):283-5. https://doi.org/10. 1136/emj.14.5.283.

20. Yeguiayan J-M, Garrigue D, Binquet C, Jacquot C, Duranteau J, Martin C, et al. Medical pre-hospital management reduces mortality in severe blunt trauma: a prospective epidemiological study. Crit Care. 2011;15(1):R34. https://doi.org/10.1186/cc9982.

21. Lerner EB, Moscati RM. The golden hour: scientific fact or medical "urban legend"? Acad Emerg Med. 2001;8(7):758-60. https://doi.org/10.1111/j.15532712.2001.tb00201.x.

22. Trunkey D. Trauma. Sci Am. 1983;249(2):20-7 Retrieved from http://www. scientificamerican.com/magazine/sa/1983/08-01/.

23. Newgard CD, Meier EN, Bulger EM, Buick J, Sheehan K, Lin S, et al. Revisiting the "golden hour": an evaluation of out-of-hospital time in shock and traumatic brain injury. Ann Emerg Med. 2015;66(1):30-41.e3. https://doi.org/ 10.1016/.annemergmed.2014.12.00.

24. Newgard CD, Schmicker RH, Hedges JR, Trickett JP, Davis DP, Bulger EM, et al. Emergency medical services intervals and survival in trauma: assessment of the "Golden hour" in a north American prospective cohort. Ann Emerg Med. 2010;55(3). https://doi.org/10.1016/j.annemergmed.2009. 07.024 .

25. Osterwalder JJ. Can the "Golden hour of shock" safely be extended in blunt Polytrauma patients? Prospective cohort study at a level I Hospital in Eastern Switzerland. Prehosp Disaster Med. 2002;17(2):75-80. https://doi.org/ 10.1017/S1049023X00000212.

26. Campbell JP, Maxey VA, Watson WA. Hawthorne effect: implications for Prehospital research. Ann Emerg Med. 1995;26(5):590-4. https://doi.org/10, 1016/S0196-0644(95)70009-9.

27. McCambridge J, Witton J, Elbourne DR. Systematic review of the Hawthorne effect: new concepts are needed to study research participation effects. J Clin Epidemiol. 2014;67(3):267-77. https://doi.org/10.1016/j.jclinepi.2013.08.015.

\section{Publisher's Note}

Springer Nature remains neutral with regard to jurisdictional claims in published maps and institutional affiliations.

Ready to submit your research? Choose BMC and benefit from:
- fast, convenient online submission
- thorough peer review by experienced researchers in your field
- rapid publication on acceptance
- support for research data, including large and complex data types
- gold Open Access which fosters wider collaboration and increased citations
- maximum visibility for your research: over 100M website views per year
At BMC, research is always in progress.
Learn more biomedcentral.com/submissions

